\title{
Determinación del caudal ambiental mediante una metodología hidrobiológica, considerando variables de cambio climático en el río Pejibaye, Cartago, Costa Rica
}

Determination of the environmental flow using a hydrobiological methodology, considering variables of climate change in the Pejibaye river, Cartago, Costa Rica

Fernando Watson-Hernández ${ }^{1}$, Laura Chavarría-Pizarro², Francisco Quesada-Alvarado ${ }^{3}$, Isabel Guzmán-Arias ${ }^{4}$

Fecha de recepción: 16 de agosto de 2020

Fecha de aprobación: 8 de diciembre de 2020

Watson-Hernández, F; Chavarría-Pizarro, L; Quesada-Alvarado, F; Guzmán-Arias, I. Determinación del caudal ambiental mediante una metodología hidrobiológica, considerando variables de cambio climático en el río Pejibaye, Cartago, costa rica. Tecnología en Marcha. Vol. 34-4 Octubre-Diciembre 2021. Pág 118-129.

doi) https://doi.org/10.18845/tm.v34i4.5223

1 Instituto Tecnológico de Costa Rica. Costa Rica. Correo electrónico: fwatson@tec.ac.cr

2 Instituto Tecnológico de Costa Rica. Costa Rica. Correo electrónico: laura.chavarria@tec.ac.cr

4 Instituto Tecnológico de Costa Rica. Costa Rica. Correo electrónico: 


\title{
Palabras clave
}

Caudal ambiental; ecohidraúlica; preferencia de hábitat; insectos acuáticos; peces.

\section{Resumen}

En Costa Rica el caudal ambiental se calcula como un 10\% del aforo caudal medio del río. Este porcentaje ha carecido de fundamento científico en relación con las condiciones fluviales aptas para preservar los ecosistemas presentes en los cauces. Las concesiones de agua para los diferentes usos han provocado un impacto ambiental que se ve reflejado en las condiciones de disponibilidad del recurso hídrico y en las condiciones fisicoquímicas y ecológicas de los cauces. Este estudio responde a la ruta de investigación que busca la selección y validación en campo de diferentes metodologías para obtener el caudal ambiental requerido en una fuente de agua superficial. En este caso, el estudio está centrado en la adaptación y validación de una metodología hidrobiológica de cálculo del régimen de caudal ambiental en la cuenca del río Pejibaye en Jiménez de Cartago. Se utilizaron series de datos históricas de variables hidrológicas para analizar la condición actual y proyectada a futuro, se seleccionaron especies de macroinvertebrados y peces que permitieran evaluar la calidad de los ecosistemas asociados, finalmente se establecieron los parámetros hidráulicos de preferencia de hábitat para definir el régimen de caudal ambiental actual, cuyos resultados revelan un comportamiento de estacionalidad, el cual se acentúa en la proyección a futuro. Se determinó que el género de insecto Anacroneuria y la especie de pez G. nudus, son los mejores para determinar caudales ambientales.

\section{Keywords}

Environmental flow; ecohydraulic; habitat preference; aquatic insects; fish.

\begin{abstract}
The environmental flow, in Costa Rica, is calculated as $10 \%$ of the average flow of the river. This percentage has lacked scientific basis in relation to the river conditions suitable for preserving the ecosystems present in the rivers. The water concessions for the different uses have caused an environmental impact that is reflected in the conditions of availability of the water resource in some vulnerable points of the country, as well as in the physicochemical and ecological conditions of the channels. The proposed project responds to the research route that seeks the selection and field validation of different methodologies to obtain the required environmental flow in a surface water source. In this particular case, the project is focused on the adaptation and validation of a hydrobiological methodology for calculating the environmental flow regime in Pejibaye river basin, Jiménez de Cartago. Historical hydrological data variables were used to analyze the current and projected future condition, the species of macroinvertebrates and fish that allowed evaluating the quality of the associated ecosystems were selected, finally, the hydraulic parameters of habitat preference were established to define the current environmental flow regime, whose results reveal a seasonality behavior, which is accentuated in the future projection. The insect genus Anacroneuria and the fish species G. nudus were determined to be the best for determining environmental flows.
\end{abstract}




\section{Introducción}

El caudal ambiental establece cuánto del régimen hidrológico natural de un río debería seguir fluyendo aguas abajo y hacia la planicie de inundación para mantener los valores característicos del ecosistema [1]. A nivel mundial, se utilizan los caudales ambientales como herramienta de la Gestión Integrada de Recursos Hídricos que permite abordar la problemática generalizada de su uso excesivo y del deterioro de los ecosistemas acuáticos [2]. El proceso de cambio climático también ha dejado en evidencia la situación crítica en la que se encuentran muchos ecosistemas acuáticos; en algunos países la situación ha empeorado tanto en los últimos años, que grandes cuerpos de agua han llegado a desaparecer, como: el Lago Mead, y el Lago Badwater en Estados Unidos; el Lago Poopó en Bolivia y el Mar Aral en Kasajistán [3].

A pesar de que el agua es un recurso imprescindible para el mantenimiento de la vida, los ecosistemas acuáticos han sufrido un proceso de degradación y sobreexplotación, que ha provocado entre otros problemas, la reducción del caudal necesario para mantener la biodiversidad y los servicios que obtienen las comunidades de éstos [4]. Costa Rica emplea, como medida para la función de estos ecosistemas un $10 \%$ del caudal de estiaje, valor que no tiene un respaldo científico y representa un modelo insostenible para una adecuada administración del recurso hídrico. Al no contar el país con un procedimiento apropiado, ha sido una prioridad de las autoridades encargadas de administrar el recurso hídrico, la implementación de una metodología donde pueda determinar el caudal ambiental necesario para mantener la funcionalidad de los ecosistemas acuáticos y sus procesos naturales (tomando en cuenta el deterioro ambiental y el proceso de cambio climático), y que además tiene un efecto directo sobre la disponibilidad del recurso para uso de las poblaciones.

Debido a que el rescate del capital hídrico, esencial para proteger y conservar las fuentes de agua, se han realizado varias iniciativas en el país para utilizar metodologías más acordes con el cálculo de los requerimientos reales [5]. Diferentes instituciones han aplicado metodologías de acuerdo con las necesidades de sus proyectos, sin embargo, no se ha logrado determinar cuál sería la más apropiada en cada caso particular; por ejemplo, algunas metodologías, dependiendo del tipo de concesión y de las características que tenga el río y su zona de influencia, podrían no ser aplicables. En el caso de la metodología holística, una de las más complejas y utilizada por el Instituto Costarricense de Electricidad (ICE), podría no ser adecuada para una valoración de aprovechamiento a muy baja escala y de un caudal pequeño. No obstante, la determinación del caudal debe ser atendida en toda su dimensión real de complejidad según las condiciones en que se encuentra la fuente y la demanda por el aprovechamiento del agua. Este estudio pretende determinar el caudal ambiental requerido para río Pejibaye, mediante la implementación de una metodología de cálculo hidrobiológica, considerando variables de cambio climático. La consecución de este objetivo se dio por medio de: establecer la respuesta hidrológica actual y futura de la cuenca del río Pejibaye ante escenarios de cambio climático; seleccionar los indicadores biológicos para el requerimiento de caudal ambiental y generar el régimen de caudal ambiental del río tomando en cuenta los componentes metodológicos establecidos y los escenarios de cambio climático.

\section{Metodología}

La cuenca del río Pejibaye está ubicada en el cantón de Jiménez en la provincia de Cartago, pertenece al sistema de la Cuenca Alta del Río Reventazón ubicada en la vertiente del caribe de Costa Rica, esta cuenta con un área aproximada de $250 \mathrm{~km}^{2}$ y un rango de altitud que va desde 570 a los 2680 m.s.n.m, presenta áreas con pendientes mayores a 60\%. Esta cuenca se encuentra cubierta por el $80 \%$ de bosque denso, el resto de la superficie presenta usos del suelo como: caña de azúcar, pastos, café y maleza. Asimismo, se caracteriza por tener 
un promedio anual de precipitación entre los 2800 y 8000 mm, en la zona centro oeste de la cuenca la precipitación puede ser de 9000 mm/año, esto provoca que sea una de las zonas más lluviosas del país, con gran potencial hidroeléctrico, registrando caudales en promedio anual de $34 \mathrm{~m}^{3} / \mathrm{s}$, haciendo un aporte importante al Proyecto Hidroeléctrico Angostura [6].

Los trayectos seleccionados para la determinación de caudales ambientales se establecieron por medio de criterios de uso de suelo, condiciones hidráulicas y biológicas, disponibilidad de datos históricos de caudales y con longitudes de entre 160 a 200 m de longitud para la obtención de representatividad de hábitats, caracterizadas hidráulicamente por la existencia de zonas con calados mayores al promedio (pozas), flujos supercríticos, subcríticos y áreas mixtas. Bajo estas condiciones, se seleccionaron tres trayectos, dos en el río Pejibaye y uno sobre el río Gato. Nombrados según el orden de recolección de muestras durante las giras de campo. El primero definido como P1 estaba en el río Pejibaye a $350 \mathrm{~m}$ al sureste de la plaza de deportes del pueblo de Pejibaye, bajo el puente que comunica con el poblado Gato, el segundo punto P2 se ubica el río Gato a $400 \mathrm{~m}$ del campo de futbol del poblado del mismo nombre y el tercer punto P3 sobre el río Pejibaye, se ubica aguas arriba del puente sobre la carretera nacional 225. Los trayectos P1, P2 y P3, para fines hidrológicos, representan los puntos de aforo de la cuenca y subcuencas en estudio; Cuenca P1, Cuenca P2 y Cuenca P3 (ver figura 1). Las cuencas P1 y P3 cuentan con datos de precipitación (P), temperatura (T) y caudal $(Q)$ para el periodo 20002009 a escala diaria, en tanto, la cuenca P2 tiene información de P y T para el mismo periodo de tiempo y misma escala temporal. La cuenca P2, al no tener datos de caudal, no se le realizó una calibración directa, sino que se utilizaron los valores de los parámetros de calibración de la cuenca P3, que es con la que presenta mayores similitudes en cuanto a distribución de uso de suelo y pendiente media de la cuenca.

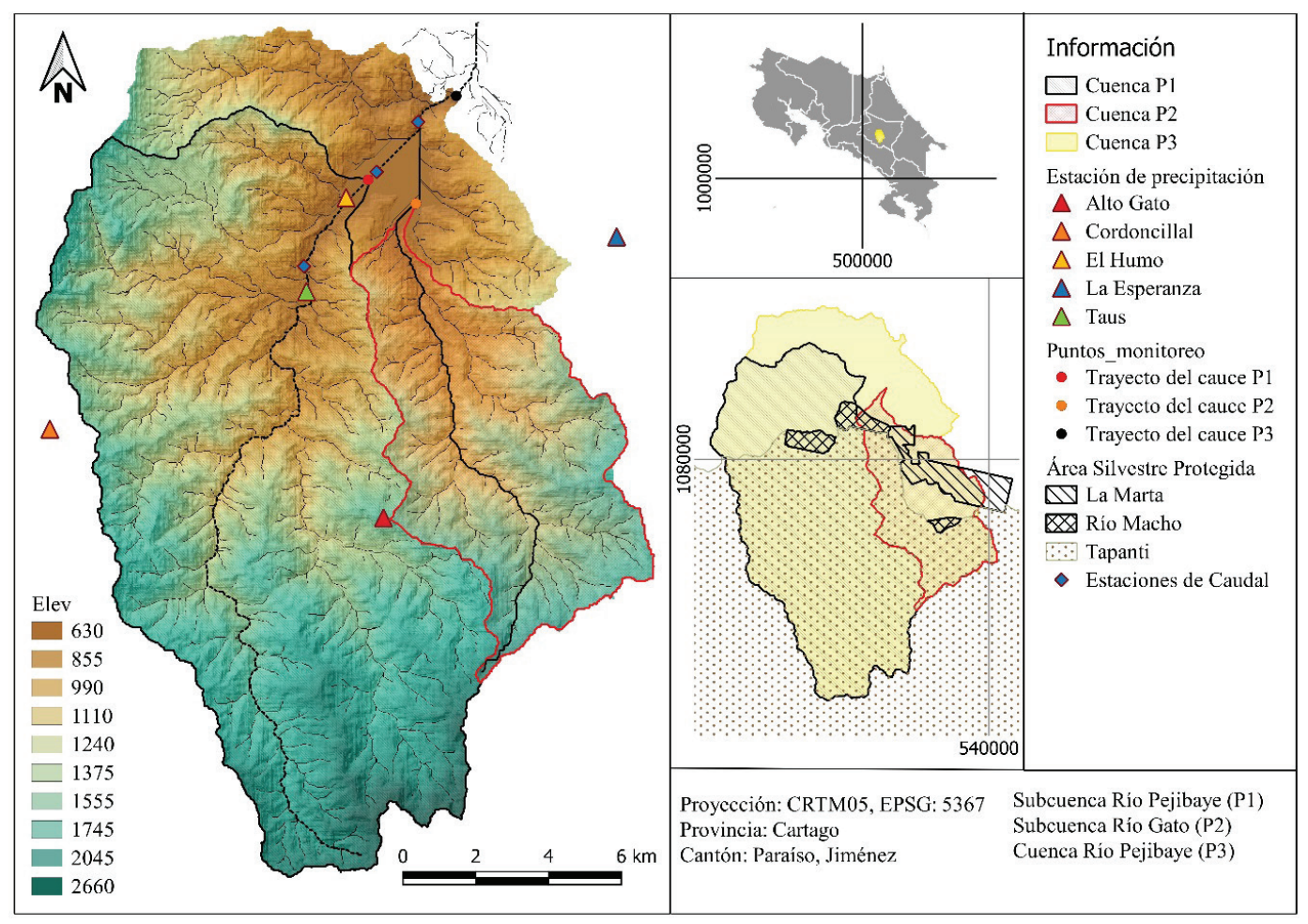

Figura 1. Ubicación de estaciones de medición (caudal y precipitación), áreas silvestres protegidas, puntos de muestreo y subdivisión de cuencas. 
Con el análisis de precipitación, temperatura atmosférica y caudales registrados, además del análisis geoespacial, se desarrolló el modelado hidrológico de las tres microcuencas correspondientes al área de influencia de los puntos seleccionados. Posteriormente se construyeron los escenarios de cambio climático con proyecciones para los periodos 20002019, 2040-2059, 2080-2099.

Se seleccionaron como organismos indicadores de caudal a insectos acuáticos y peces. Estos organismos fueron seleccionados ya que están adaptados a las fluctuaciones naturales en magnitud, frecuencia, y duración de las corrientes de flujo (alto, intermedio o bajo). Específicamente, para macroinvertebrados acuáticos se tomaron muestras de ninfas de los órdenes de insectos Ephemeroptera (Baetidae; Baetodes sp.) y Plecoptera (Perlidae; Anacroneuria sp.).

En cuanto a los peces como indicadores de caudal, para este estudio se utilizó a las especies Gobiexus nudus, Rhamdia laticauda y Astyanax aeneus. En el caso de Gobiexus nudus, es una especie que suele habitar en los sistemas tipo rápidos y turbulentos, por lo que puede ser un buen indicador de caudal, debido a que los rápidos y las cataratas son los microhábitats más perjudicados al reducir el caudal. Rhamdia y Astyanax, se han registrado en distintos microhábitats determinados por la velocidad del agua, sin embargo, en el caso de Astyanax son más frecuentes en pozas y aguas calmas. Aun así, es importante corroborar su eficacia como especies indicadoras de caudal ambiental.

Luego, se elaboraron las curvas de preferencia de hábitat y se estableció tres categorías de preferencia: hábitat óptimo, es el rango donde se encuentra la mayor abundancia de organismos; regular, son el conjunto de rangos donde hay presencia de organismos, pero en menor abundancia; inadecuado, es el rango (os) donde no hay presencia del organismo objetivo. Posteriormente, por medio del software Iber 2.5.1, se realizaron simulaciones de hábitat, que son modelos que tienen como objetivo evaluar la relación del caudal con la formación de hábitat físico óptimo, regular o inadecuado para las especies objetivo, y de esta forma evaluar los efectos del cambio de caudal. Para realizar la simulación se utilizaron los datos obtenidos de los indicadores biológicos y los parámetros hidrológicos: profundidad y velocidad del agua. Finalmente, se determinó el régimen de caudal ambiental para cada uno de los tres puntos de estudio, según los criterios establecidos para establecer las características del caudal óptimo.

\section{Resultados}

Por medio de la simulación de hábitat, se generaron las curvas de área utilizable ponderada (WUA) en unidades porcentuales del área del espejo de agua. La variación de WUA está en función del caudal sobre el cauce. Estas curvas se obtuvieron para los trayectos de cauce de las tres cuencas para las especies Anacroneuria, Astyanax aeneus, Baetodes, Gobiexos nudus y Rhamdia laticauda. A manera de ejemplo, se muestra el análisis en el punto P3, el cual muestra al igual que en el cauce de la cuenca P1 y P2, que la especie Baetodes tiene la mayor área de hábitat útil (ver figura 2), alcanzando un máximo de 93,5\% del espejo de agua para un flujo de $10 \mathrm{~m} 3 / \mathrm{s}$. La curva de Astyanax aeneus igualmente, es la segunda que mayor área de hábitat utilizable presenta con un $75 \%$ del espejo de agua para para un caudal de 2 m³/s. La especie que presenta un menor pico es la Rhanmdia laticauda con un hábitat máximo de 53\% para un flujo de $4 \mathrm{~m}^{3} / \mathrm{s}$. 


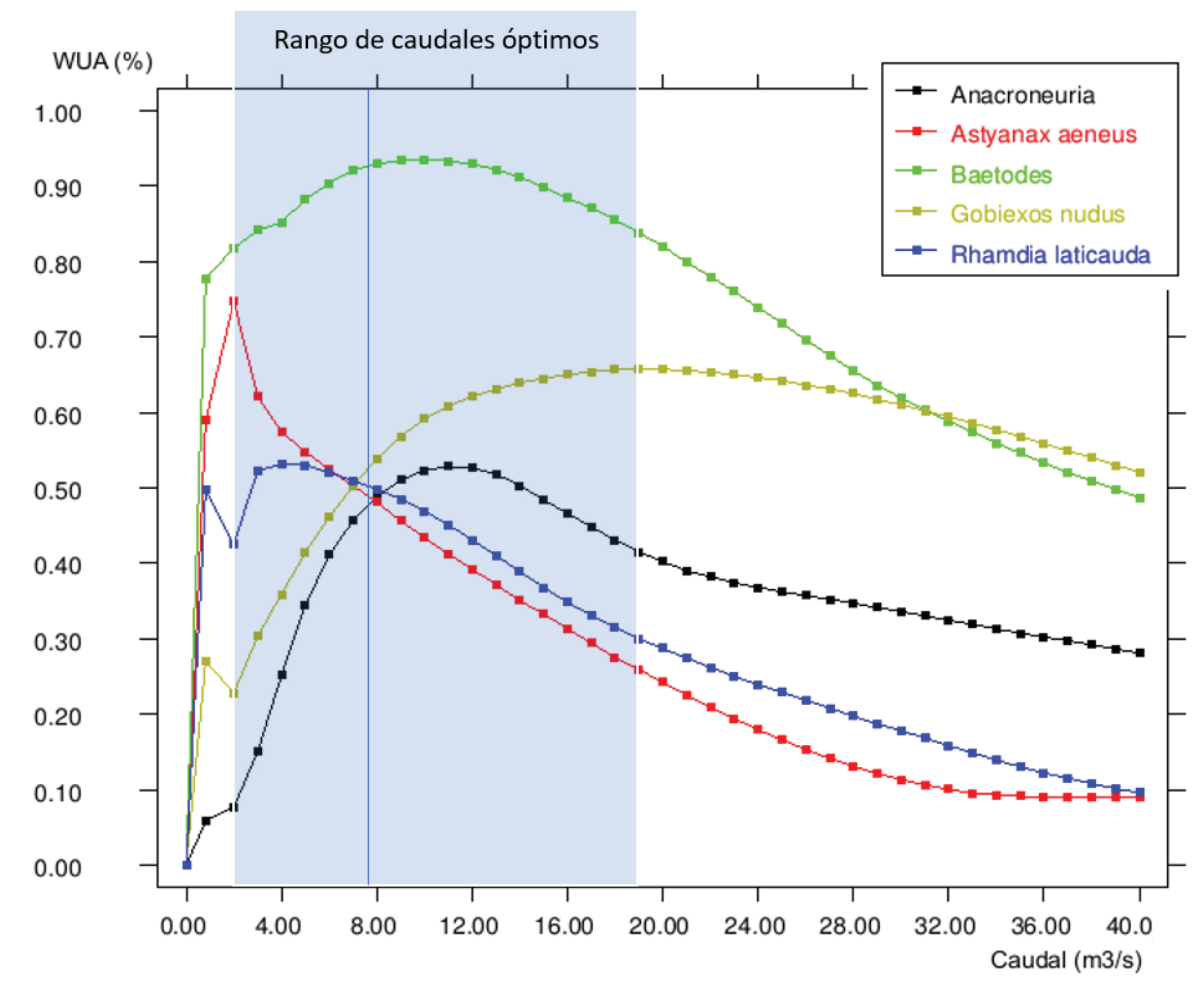

Figura 2. Comportamiento del Área utilizable ponderada (WUA\%) en respuesta del caudal en el cauce de la cuenca P3 para las 5 especies indicadoras.

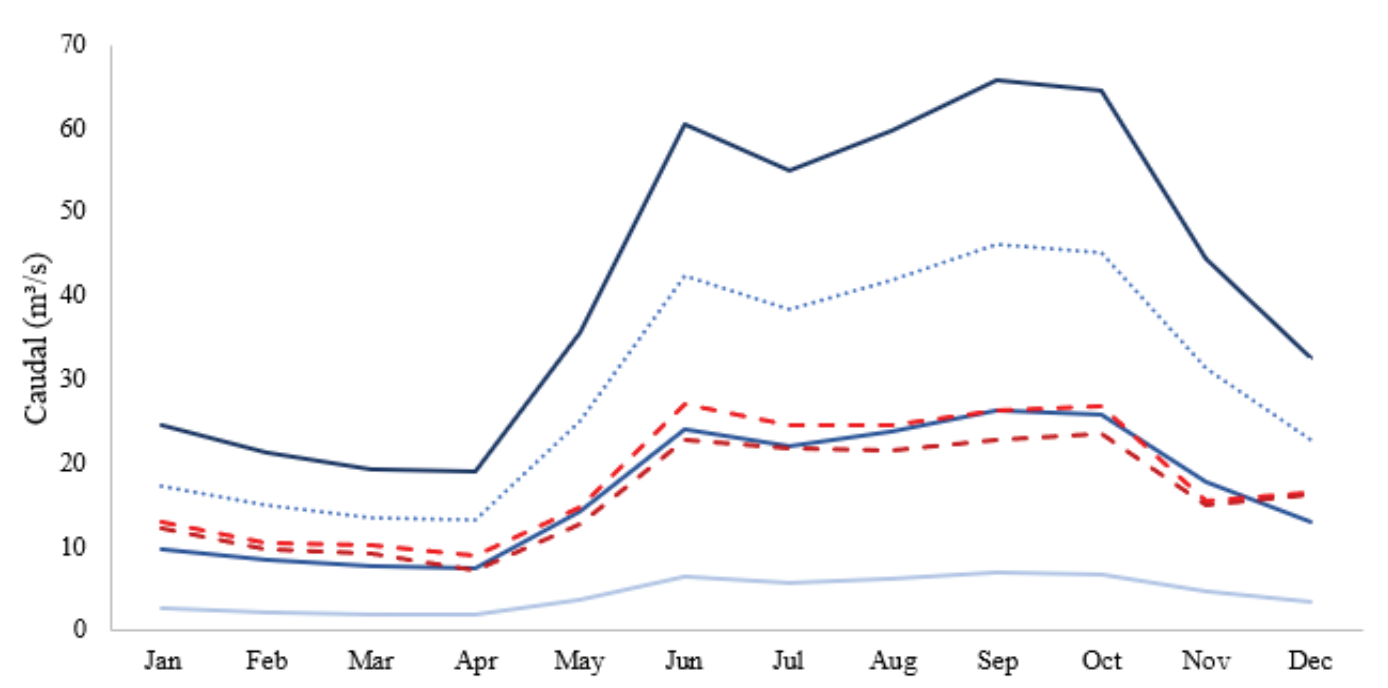

....... Q promedio mensual 2000-2019 Límite Inferior de caudal óptimo según WUA

$\begin{array}{ll}\text { - Valor medio de caudal óptimo según WUA } & \text { - Limite superior de caudal óptimo según WUA } \\ \text { - - Caudal de Excedencia } 90 \% & \text { - Caudal de Excedencia } 95 \%\end{array}$

Figura 3. Resumen de las propuestas para establecer el régimen de caudal ambiental. 
El rango de caudales óptimo es el más amplio del estudio, con caudales entre 2 a 19 m³/s. Aplicando la misma metodología, se establece un valor medio 7,6 $\mathrm{m}^{3} / \mathrm{s}$ (ver figura 3). De igual forma, el caudal más bajo se presenta en abril con 13,33 $\mathrm{m}^{3} / \mathrm{s}$, los valores de caudal óptimos de 2, 7,6 y 19 m³/s. Los porcentajes de disminución son del 85\%, 43\% para límite medio e inferior, de igual forma, esta cuenca tiene un límite superior de caudal óptimo mayor que el caudal medio del mes de abril. En este caso se tomó el límite medio para establecer la recomendación del régimen de caudales, según se muestra en la figura 4.

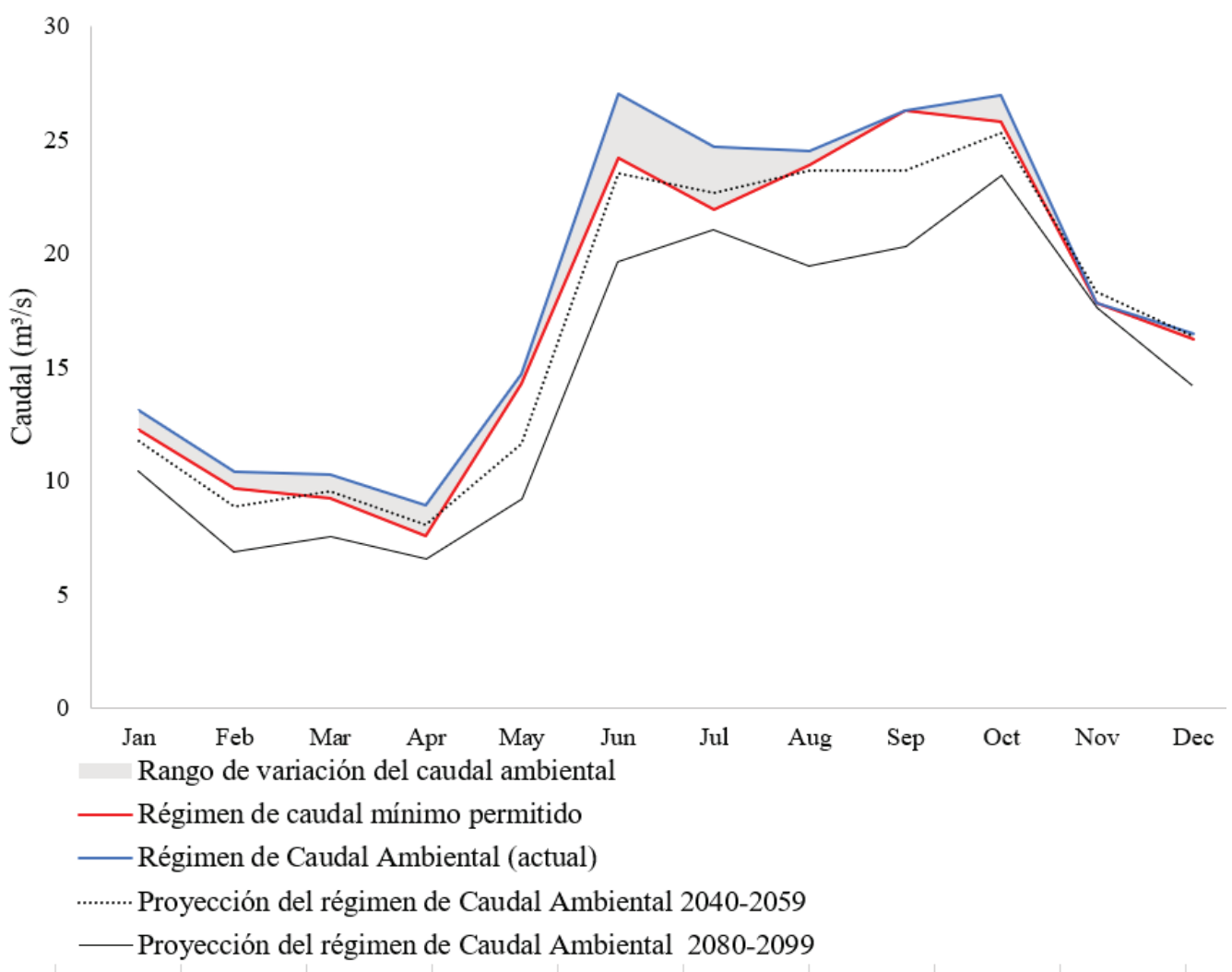

Figura 4. Régimen de caudal ambiental recomendado para la cuenca P3.

Al simular este régimen de flujo recomendado se obtiene que Baetodes en los primeros 4 meses del año presenta valores de WUA del 93\%, el valor mínimo lo presenta en octubre con un $68 \%$ y el promedio anual está en el $82 \%$ del espejo de agua. Astyanax aeneus presenta el valor promedio anual menor de $29 \%$, presentando su máximo del $46 \%$ en abril. En el cuadro 1 se detallan los valores de WUA calculados para cada una de las especies en función de los caudales del régimen hídrico recomendado, producto de la simulación espacial del comportamiento de las áreas utilizables para cada especie. 
Cuadro 1. Comportamiento del área utilizable ponderada en respuesta del régimen de caudal ambiental recomendado.

\begin{tabular}{|c|c|c|c|c|c|c|}
\hline Mes & Caudal & $\begin{array}{c}\text { WUA } \\
\text { Gobiexos } \\
\text { nudus }(\%)\end{array}$ & $\begin{array}{c}\text { WUA } \\
\text { Astyanax } \\
\text { aeneus }(\%)\end{array}$ & $\begin{array}{c}\text { Rhamdia } \\
\text { laticauda } \\
(\%)\end{array}$ & $\begin{array}{c}\text { WUA } \\
\text { Anacroneuria } \\
(\%)\end{array}$ & $\begin{array}{c}\text { WUA } \\
\text { Baetodes } \\
(\%)\end{array}$ \\
\hline Jan & 13,1 & $63 \%$ & $37 \%$ & $41 \%$ & $52 \%$ & $92 \%$ \\
\hline Feb & 10,4 & $60 \%$ & $43 \%$ & $46 \%$ & $53 \%$ & $94 \%$ \\
\hline Mar & 10,3 & $60 \%$ & $43 \%$ & $46 \%$ & $53 \%$ & $94 \%$ \\
\hline Apr & 8,9 & $57 \%$ & $46 \%$ & $49 \%$ & $51 \%$ & $93 \%$ \\
\hline May & 14,7 & $64 \%$ & $34 \%$ & $37 \%$ & $49 \%$ & $90 \%$ \\
\hline Jun & 27,0 & $63 \%$ & $14 \%$ & $21 \%$ & $35 \%$ & $68 \%$ \\
\hline Jul & 24,7 & $64 \%$ & $17 \%$ & $23 \%$ & $36 \%$ & $72 \%$ \\
\hline Aug & 24,5 & $64 \%$ & $17 \%$ & $23 \%$ & $37 \%$ & $73 \%$ \\
\hline Sep & 26,3 & $64 \%$ & $15 \%$ & $22 \%$ & $36 \%$ & $69 \%$ \\
\hline Oct & 27,0 & $63 \%$ & $14 \%$ & $21 \%$ & $35 \%$ & $68 \%$ \\
\hline Nov & 15,4 & $65 \%$ & $33 \%$ & $36 \%$ & $48 \%$ & $89 \%$ \\
\hline Dec & 16,5 & $65 \%$ & $30 \%$ & $34 \%$ & $46 \%$ & $88 \%$ \\
\hline Máximo & & $19,0 / 66 \%$ & $2,0 / 75 \%$ & $4,0 / 53 \%$ & $11,0 / 53 \%$ & $10,0 / 94 \%$ \\
\hline Caudal/WUA & & & & & & \\
\hline
\end{tabular}

\section{Discusión}

\section{Requerimiento del caudal ambiental según indicadores biológicos}

El flujo de agua tiene una gran influencia en el establecimiento de los organismos acuáticos, ya que afecta la composición del sustrato, las propiedades químicas del agua, el movimiento de nutrientes y partículas orgánicas, así como la disponibilidad e idoneidad del hábitat [7]. Las actividades humanas podrían extender los períodos de caudal reducido a lo largo del año; por este motivo, es importante identificar aquellos organismos con requerimientos de caudal más estrictos, para garantizar un flujo que permita su supervivencia y la de especies asociadas. En el caso del río Pejibaye, se encontraron organismos bioindicadores que permitirían la determinación del caudal ambiental como se muestra a continuación.

\section{Insectos}

A pesar que Baetodes fue encontrado en un rango mayor de hábitats, al igual que Anacroneuria fue encontrado en hábitats no muy profundos y con corriente, esto se debe a que ambas especies presentan adaptaciones para establecerse en zonas con corriente como un cuerpo aplanado y alargado, así como uñas para adherirse a los sustratos [8]. De acuerdo con Brown \& Brussock [9] los efemerópteros como Baetodes tienen adaptaciones para habitar diferentes rangos de velocidad y caudal, por este motivo posiblemente no presentaron una preferencia particular de hábitat.

En general, ambas especies no fueron encontradas en hábitats con valores muy altos y bajos de caudal. En el caso de un caudal alto, podría desfavorecer la presencia de estos insectos al provocar el arrastre de sedimentos como hojarasca, cantos rodados y piedras que ofrecen refugio y alimento para los individuos de ambas especies (de Baetodes como raspador, y Anacroneuria como depredador de larvas de otros insectos) [10] [11]. Por otra parte, un caudal 
bajo (natural o artificial) también tiende a reducir la densidad de macroinvertebrados en los cuerpos de agua [12] [13]. Esta reducción en la densidad, se da como respuesta a un aumento en las dinámicas de competencia y depredación, ya que, al disminuir la cantidad de hábitat, disminuye la cantidad y calidad del alimento [12].

Según los resultados obtenidos se puede concluir que Baetodes es más generalista y puede habitar un rango mayor de hábitats, mientras que Anacroneuria es más selectivo y presenta preferencia por hábitats con características más específicas, sugiriendo que ésta especie podría ser un buen indicador en la determinación del régimen de caudal cuando estuviera presente en el cuerpo de agua estudiado. Adicionalmente, ambas especies fueron encontradas en diferentes épocas del año lo que favorece la utilización en este tipo de organismos en este tipo de estudios.

\section{Ictiofauna}

Para las tres especies de peces evaluadas en el río Pejibaye, se observó que, al aumentar la profundidad y velocidad del agua, aumentó la cantidad de organismos, sin embargo, se llegó a un punto de quiebre y la abundancia empezó a disminuir. De igual forma, al aumentar el caudal aumentó el porcentaje de hábitat utilizable, pero el aumento continuo del caudal provocó la disminución en la cantidad de hábitat disponible. Estas condiciones obedecen a que los organismos cuentan con adaptaciones morfológicas que les permiten establecerse en uno o varios tipos de microhábitats, pero ante condiciones extremas se empieza a registrar menor cantidad de organismos [14].

La especie Gobiesox nudus, cuenta con una ventosa ventral y cuerpo aplanado dorsoventralmente, que le permite mantenerse en las zonas con mayor velocidad y turbulencia y de esta forma evitar depredadores [15]. No obstante, a velocidades mayores a los 1,2 m/s es posible que la especie experimente mayor dificultad para mantenerse o desplazarse esto debido a la incidencia del número de Reynolds por su tamaño [16] y de ahí se dé una disminución en su frecuencia, también considerando que los organismos evitan los sitios donde pierden más energía de la que adquieren alimentándose [14]. Para Rhamdia laticauda y Astyanax aeneus, la mayor abundancia se dio a velocidades que van desde los $0 \mathrm{~m} / \mathrm{s}$ hasta los 0,6 m/s; esta condición de no estar presentes en mayor abundancia a velocidades extremas puede estar medida por la forma corporal y hábitos alimenticios de cada especie [17]. La posición de la boca de $A$. aeneus, le permite picar el fondo y superficie del agua para cazar insectos y frutos, y por su forma corporal ovoide [15], le dificultan permanecer en zonas con mayor fuerza de arrastre. Por su parte, R. laticauda, su boca se encuentra direccionada de forma frontoventral, permitiéndole alimentarse del fondo del río, por lo que se puede registrar con mayor frecuencia en zonas lentas [15]. Sin embargo, su cuerpo de forma de torpedo, le permite de igual forma movilizarse y asentarse en hábitats de mayor velocidad [18], pero siempre protegiéndose u ocultándose entre las piedras cercanas al fondo, donde la velocidad es menor [19].

En el caso de estas tres especies de peces el rango de hábitat y las curvas de preferencia, no pueden ser comparadas con otros estudios, ya que no hay registro bibliográfico sobre este tema para estas especies. No obstante, [20] en su estudio determinaron la relación entre la forma del cuerpo y los aspectos ecológicos con respecto al uso del hábitat, utilizando varias especies de talla media, entre ellas el género Astyanax sp y la especie Imparfinis minutus (familia Heptapteridae, al igual que $R$. laticauda). En su estudio observaron que la mayor abundancia de Astyanax sp. se dió entre las velocidades de 0,5 y 1,0 m/s y en el rango de profundidad entre los 40 y $60 \mathrm{~cm}$, similar a la especie costarricense $A$. aeneus. Mientras que I. minutos fue registrada con mayor abundancia entre las velocidades entre 1,5 y 2,0 m/s, diferente a $R$. laticauda la cual prefirió velocidades menores, sin embargo, esta disimilitud puede deberse a que a pesar de que pertenecen a la misma familia y con características morfológicas similares, tienen distintas 
preferencias por la velocidad del agua, o bien, porque durante en varios muestreos en el río Pejibaye no se pudo utilizar la electropesca dificultando así la observación en los macrohábitats turbulentos. En cuanto a la profundidad, I. minutos la registraron entre 10 y $20 \mathrm{~cm}$, mientras que $R$. laticauda fue registrada con mayor abundancia entre 40 y $50 \mathrm{~cm}$, por lo que para esta variable también hay diferencias.

Por otro lado, [21] obtuvieron curvas de preferencia de hábitat para cinco especies de peces, todas con cuerpo en forma de torpedo y de tamaño pequeño, y observaron la misma tendencia que en el río Pejibaye, donde las especies logran utilizar velocidades nulas y de corriente moderada a fuerte, pero menores a los $1 \mathrm{~m} / \mathrm{s}$. Aun así, la presencia de una especie en un tipo de hábitat no solo puede estar medida por la forma corporal [20], sino también por sus hábitos alimenticios y, por lo tanto, donde se encuentre las presas que consumen con mayor frecuencia [22]. A la vez, en el caso de la ictiofauna se recomienda a la especie G. nudus como la mejor especie indicadora de caudal ambiental, ya que cuentan con menores rangos de velocidad y profundidad consideradas como óptimas, por lo que variaciones con tendencia a la disminución pueden representar la disminución de sus respectivos hábitats idóneos y como lo menciona [23], ante disminución de caudal los hábitats más perjudicados son los rápidos y las cataratas, poniendo en riesgo a las especies reófilas.

\section{Caudal ambiental}

Muchas investigaciones han combinado los modelos ecológicos e hidrodinámicos para determinar la relación entre el caudal y la preferencia del hábitat físico en especies acuáticas. Las curvas de preferencia de hábitat, han sido usadas para caracterizar la preferencia de hábitat y la disponibilidad y calidad del hábitat de especies acuáticas [24]. Así, a través de modelos de cuantificación del porcentaje de hábitat idóneo para las especies acuáticas según un caudal, se puede determinar bajo cuál o cuáles volúmenes de agua se puede mantener las condiciones requeridas por una o varias especies en aquellos ríos donde haya permisos de extracción de agua. En el caso del río Pejibaye, con las curvas de área utilizable ponderada o porcentaje de hábitat idóneo para las especies acuáticas en el sitio P1, se pudo determinar que caudales entre los 1 y $4 \mathrm{~m}^{3} / \mathrm{s}$, fue donde se genera la mayor cantidad de hábitat disponible para estas especies, y que durante la época seca o de estiaje para este tramo el caudal ambiental mínimo aceptable sería de $4 \mathrm{~m}^{3} / \mathrm{s}$. Para el sitio P2, caudales entre los 1 y $5,5 \mathrm{~m}^{3} / \mathrm{s}$, generan la mayor cantidad de hábitat disponible (más del 50\%) para las cinco especies acuáticas estudiadas, con un caudal ambiental mínimo aceptable de $1 \mathrm{~m} 3 / \mathrm{s}$. Por último, el sitio P3 la mayor cantidad de hábitat disponible para las especies estudiadas se da entre 2 y $18 \mathrm{~m}^{3} / \mathrm{s}$, y bajo un caudal de $8 \mathrm{~m}^{3} / \mathrm{s}$ es donde la mayoría de las especies cuentan con más de un $50 \%$ de hábitat idóneo. El caudal ambiental mínimo aceptable para este tramo cercano a los $8 \mathrm{~m}^{3} / \mathrm{s}$.

Sin embargo, se debe recalcar que el caudal mínimo aceptable no es solo para la época de estiaje, sino que durante la época lluviosa también se determina un caudal mínimo aceptable para cada tramo del río Pejibaye. Por lo que también se maneja el término de caudal ambiental adaptativo, que es aquel que permite cambios durante todo el año para dar mantenimiento al río, las especies acuáticas y todos los procesos ecológicos propios del ecosistema acuático. De esta manera en la época lluviosa, para el sitio P1 el rango de caudal ambiental es de 4,2 a $4 \mathrm{~m}^{3} / \mathrm{s}$, para el sitio P2 es de 1,45 a 1,4 m³/s y para el sitio P3 sería de 8,9 a 7,6 m³/s. el caudal ambiental puede variar en ese rango sin que represente una amenaza al ecosistema. En la época lluviosa pueden estar variando constantemente a valores mayores. 
En una proyección de cambio climático a los periodos 2040 - 2059 y 2080 - 2099, el caudal ambiental se establece en 4,14 y 3,68 $\mathrm{m}^{3} / \mathrm{s}$ respectivamente para $\mathrm{P} 1$, en 1,37 y 1,09 $\mathrm{m}^{3} / \mathrm{s}$ para P2 y en 8,08 y $6,56 \mathrm{~m}^{3} / \mathrm{s}$ para P3. En estos escenarios, los caudales se acercan o sobrepasan en algunos casos, al límite inferior actual, lo cual evidencia el impacto en el régimen disponible de caudal en el río.

\section{Conclusiones y Recomendaciones}

La respuesta hidrológica actual y futura de la cuenca del río Pejibaye ante escenarios de cambio climático, evidencia un comportamiento de estacionalidad en la actualidad, el cual se acentúa en la proyección a futuro conforme se extiende el periodo de tiempo proyectado, así lo demuestran las curvas de excedencia del periodo base 2000-2019 con los periodos 2040-2059 y 2080-2099, donde se obtuvo que el primer trimestre es el que presenta una mayor afectación relativa.

Por su menor rango en la selección de hábitat óptimo, el género de insecto Anacroneuria y la especie de pez G. nudus, son los mejores para determinar caudales ambientales.

El utilizar un conjunto de especies acuáticas para determinar sus preferencias de hábitat puede ser más efectivo para determinar caudales ambientales, ya que se puede observar los distintos tipos de requerimientos y bajo a cuáles caudales se obtienen.

Según las preferencias de hábitat y el porcentaje de hábitat idóneo para cada especie acuática, en época de estiaje (abril) se recomienda un caudal de $4 \mathrm{~m}^{3} / \mathrm{s}$ para el sitio $\mathrm{P} 1,1 \mathrm{~m}^{3} / \mathrm{s}$ en el sitio P2 y $8 \mathrm{~m}^{3} / \mathrm{s}$ en P3.

Estos caudales representan un factor del 0,43, 0,56 y 0,57 del caudal de excedencia del 50\% para el mes de abril. Utilizando estos mismos coeficientes aplicados a los demás meses del año y con la condición estar entre los caudales de excedencia del 90 al 95\% se establecieron los regímenes de caudal ambiental en las tres cuencas.

Bajo el escenario de cambio climático, se determinó que al aplicar la misma metodología para el periodo 2040-2059, las cuencas en el primer semestre del año presentarán caudales inferiores al mínimo permitido del periodo actual y en el periodo 2080-2099 esta condición se produce en todo el año.

\section{Referencias}

[1] R.E. Tharme, «A Global Perspective on Environmental Flow Assessment: Emerging Trends in the Development and Application of Environmental Flow Methodologies for Rivers, » River Res. Appl, vol. 19, pp. 397-441, 2003.

[2] M. Dyson, G. Bergkamp, y J. Scanlon, «Caudal. Elementos esenciales de los caudales ambientales». UICNORMA San José CR, 2003, pp xiv+125.

[3] "8 ríos y lagos que se están secando por el calentamiento global", Ecoosfera, 2016. [En línea]. Disponible en: http://ecoosfera.com/2016/02/8-rios-y-lagos-que-se-estan-secando-por-el-calentamiento-global/. [Accedido: 30 Mayo 2020]

[4] Guzmán-Arias, I; Watson, F; Villagran-Mendoza, K. Determinación del caudal ambiental en el río Birrís, Costa Rica. Tecnología en Marcha. Vol. 32-4. Octubre-Diciembre 2019. Pág 18-27, 2019

[5] J. Calvo Alvarado, J. A. Jiménez, E. González, F. Pizarro, and A. Jiménez, "Determinación preliminar del caudal ambiental en el río Tempisque , Costa Rica: el enfoque hidrológico con limitación de datos," Kurú, vol. 5, no. 13, pp. 1-18, 2008

[6] Zuleta, C. Estimación de los efectos del cambio climático y el cambio en el uso del suelo en el caudal de la cuenca del río Pejibaye-Costa Rica, mediante el modelo hidrológico Swat. Análisis del comportamiento del recurso hídrico ante cambios en el uso del suelo y el cambio climático en la cuenca del río Pejibaye, Costa Rica, 21-67, 2013 
[7] Z.S. Dewson, A.B.W. James, y R.G. Death, «A review of the consequences of decreased flow for instream habitat and macroinvertebrates, "Journal of the North American Benthological Society, vol 26, no. 3, pp. 401-415, 2007.

[8] D. Vásquez, R.W. Flowers, y M. Springer, «Life history of five small minnow mayflies (Ephemeroptera: Baetidae) in a small tropical stream on the Caribbean slope of Costa Rica, » Aquatic Insects: International Journal of Freshwater Entomology, vol. 31, no 1, pp. 319-332, 2009.

[9] A.V. Brown, y P.P. BrussocK, «Comparisons of benthic invertebrates between riffles and pools, » Hydrobiologia, vol. 220, pp. 99-108. 1991.

[10] D. F. Buss, D. F. Baptista, J.L. Nessimian, y M. Egler, «Substrate specificity, environmental degradation and disturbance structuring macroinvertebrate assemblages in neotropical streams,» Hydrobiologia, vol. 518, no. 1, pp. 179-188, 2004.

[11] M.P. Silveira, D.F. Buss, J.L. Nessimian, y D.F. Baptista, «Spatial and temporal

distribution of benthic macroinvertebrates in a Southeastern Brazilian river,» Brazilian Journal of Biology, vol. 66, pp. 623-632. 2006.

[12] M.D. McIntosh, M.F. Benbow, y A. J. Burky, «Effects of stream diversion on riffle macroinvertebrate communities in a Maui, Hawaii, stream, " River Research and Applications, vol. 18, pp. 569-581. 2002.

[13] R. A. I. Kinzie, C. Chong, J. Devrell, D. Lindstrom, y R. Wolff, «Effects of water removal on a Hawaiian stream ecosystem, » Pacific Science, vol. 60, pp. 1-47, 2006.

[14] P. Parasiewicz y M.J. Dunbar. "Physical habitat modelling for fish - a developing approach. Large Rivers. 12 (2-4), 239-268. 2001.

[15] W. Bussing. "Peces de las aguas continentales de Costa Rica". Editorial Universidad de Costa Rica, San Pedro, Costa Rica.468 pp, 1998.

[16] Baumgart, J. \& Friedrich, B. Swimming across scale. Nature Physics, 10 (10), 758-761, 2014.

[17] F. Quesada-Alvarado, y F. Campos. "Morphometry and burst swimming in six continental fish species from Costa Rica". Cuadernos de investigación, 11(3), 395-402, 2019.

[18] N. Gordon, T. McMahon y B. Finlayson. "Stream Hydrology An Introduction for Ecologists". John Wiley \& Sons Ltd, The Atrium, Southern Gate, Chichester, West Sussex PO19 8SQ, England. 2004.

[19] H.B. Hynes. "The ecology of running waters". Liverpool University Press, Liverpool, U.K. 1970.

[20] C. Gontijo, N. Tadini, y P. Santos. "Morphology and habitat use by fishes of the Rio das Velhas basin in southeastern Brazil". Environmental Biology of Fishes, 90 (1), 143-157. 2011.

[21] I. Dongkyun, C. Sung-Uk, y C. Byungwoong. "Physical habitat simulation for a fish community using the ANFIS method". Ecological Informatics, 43, 73-83. 2018.

[22] K. Seung y C. Sung. "Prediction of suitable feeding habitat for fishes in a stream using physical habitat simulations". Ecological Modelling, 385 (1), 65-77. 2018.

[23] R. M. Cortes, M. T. Ferreira, S. V. Oliveira, y D. Oliveira. "Macroinvertebrate community structure in a regulated river segment with different flow conditions. River Research and Applications". 18(4), 367-382. 2002.

[24] F. Wang y B. Lin. "Modelling habitat suitability for fish in the fluvial and lacustrine regions of a new Eco-City". Ecological Modelling, 267, 115-126. 2013. 